\title{
An Anionic Polymer Incorporating Low Amounts of Hydrophobic Residues Is a Multifunctional Surfactant. Part 2: Emulsification, Moisture Absorption, and Moisture Retention of Alkyl Esterified Poly- $\gamma$-Glutamic Acid
}

\author{
Makoto Urai1,2*, Tomoko Aizawa1, Mutsuyasu Nakajima', Michio Sunairi' \\ ${ }^{1}$ Laboratory of Molecular Microbiology, Department of Applied Biological Science, College of Bioresource \\ Sciences, Nihon University, Fujisawa, Japan \\ ${ }^{2}$ Department of Chemotherapy and Mycoses, National Institute of Infectious Diseases, Tokyo, Japan \\ Email: urai@niid.go.jp
}

Received 25 February 2015; accepted 27 March 2015; published 1 April 2015

Copyright @ 2015 by authors and Scientific Research Publishing Inc. This work is licensed under the Creative Commons Attribution International License (CC BY). http://creativecommons.org/licenses/by/4.0/ (c) (i) Open Access

\section{Abstract}

In the accompanying paper (Part 1), we showed that the fatty acid moiety and the acidity of the polysaccharide-backbone of a fatty acid-containing polysaccharide might be involved in its emulsification, moisture absorption, and moisture retention abilities. In this study, we synthesized alkyl esterified poly- $\gamma$-glutamic acid (PGA) with various chain lengths and degrees of substitution of the alkyl moieties to examine how hydrophobic groups incorporated in the anionic polymer contribute to enhanced emulsification, moisture absorption, and moisture retention. With a low degree of alkylation of PGA, these abilities were drastically improved. To improve the moisture absorption of PGA, alkylation with a short chain length is effective in forming interspaces between PGA chains to trap water molecules. Hydrophobic-hydrophilic balance may also be important to improve the emulsification and moisture retention abilities of PGA alkylates. To the best of our knowledge, this is the first report of the relationship between the structure and the multifunctional abilities of an anionic polymer incorporated with a small amount of hydrophobic residue. PGA alkylates, as well as fatty acid-containing polysaccharides, have potential use as multifunctional surfactants throughout various industries.

\footnotetext{
${ }^{*}$ Corresponding author.
}

How to cite this paper: Urai, M., et al. (2015) An Anionic Polymer Incorporating Low Amounts of Hydrophobic Residues Is a Multifunctional Surfactant. Part 2: Emulsification, Moisture Absorption, and Moisture Retention of Alkyl Esterified Poly- $\gamma$ Glutamic Acid. Advances in Chemical Engineering and Science, 5, 181-191. http://dx.doi.org/10.4236/aces.2015.52020 
Keywords

Poly- $\gamma$-Glutamic Acid (PGA), Alkyl Ester, Emulsification, Moisture Absorption, Moisture Retention

\section{Introduction}

We have been studying the functions and structures of extracellular polysaccharides (EPS) produced by several Rhodococcus species [1]-[8]. In the accompanying paper (Part 1), we examined the effect of structure on emulsification, thickening, moisture absorption, and moisture retention of the fatty-acid-containing extracellular polysaccharide (FACEPS) produced by Rhodococcus, and suggested that the fatty acid moiety of FACEPS was involved in these abilities. Simultaneously, we demonstrated that the emulsification, moisture absorption, and moisture retention of commercially available anionic polysaccharides, such as carboxymethyl cellulose, were improved by palmitoylation, suggesting that the acidity of the polysaccharide backbone was also involved. These observations are useful for creating new multifunctional surfactants for various industries. To understand how hydrophobic groups incorporated in the anionic polymer contribute to improving emulsification, moisture absorption, and moisture retention, it is necessary to synthesize a polymer having a more simplified structure than that of the polysaccharide.

Poly- $\gamma$-glutamic acid (PGA) is an anionic and high-molecular-weight biopolymer produced abundantly by several bacterial strains, such as Bacillus spp [9]. Owing to its biodegradability and biocompatibility, PGA has been applied to cosmetic and pharmaceutical materials [9]. Recently, chemical modifications of PGA were attempted to create materials with greater functionality [10]. One of the benefits of using PGA to create new materials is its simple structure, which is useful for understanding the relationship between structure and function. We speculate that PGA incorporated with a small amount of alkyl esters will have similar properties to those of the rhodococcal FACEPS, such as improved emulsification, moisture absorption, and moisture retention. In this paper, we synthesized alkyl esterified PGAs with various alkyl chain lengths and degrees of substitution to examine the effect of alkyl ester on the emulsification, moisture absorption and moisture retention abilities of the anionic biopolymer.

\section{Materials and Methods}

\subsection{Materials}

Poly- $\gamma$-glutamic acid (average molecular weight of $2-5 \times 10^{5}$ ), clove oil, glycerol, silica gel, and urea were obtained from Wako Pure Chemical Industries, Ltd. (Kyoto, Japan). Sodium cholate and gelatin were obtained from Dojindo Molecular Technologies, Inc. (Kumamoto, Japan) and Difco laboratories, respectively.

\subsection{Preparation of PGA Alkylates}

PGA alkylates were prepared as described by Kubota et al. with some modifications [11]. PGA (323 mg) and $\mathrm{NaHCO}_{3}(210 \mathrm{mg}$ ) were dissolved in $15 \mathrm{ml}$ of dimethyl sulfoxide (DMSO). Various concentrations of hexyl, dodecyl, or hexadecyl bromide were then added to this solution and stirred for $19 \mathrm{~h} \mathrm{at} 60^{\circ} \mathrm{C}$. The reaction mixture was extensively dialyzed against MilliQ water, and then lyophilized. The products were dissolved in DMSO- $d_{6}$ and analyzed by NMR to confirm their alkylation and to calculate the degree of alkylation. All NMR spectra were recorded at $500 \mathrm{MHz}\left({ }^{1} \mathrm{H}\right)$ and $125 \mathrm{MHz}\left({ }^{13} \mathrm{C}\right)$ with an ECA 500 instrument (Jeol Ltd. Tokyo, Japan). Assignment of signals was made based on double quantum filtered-correlation spectroscopy, heteronuclear multiple-quantum coherence, and heteronuclear multiple-bond coherence spectra. The degree of alkylation was calculated by the intensity ratio of ${ }^{1} \mathrm{H}$ NMR signals of methyl protons in the alkyl chains (A) and all methylene protons in the PGA (B) using the following equation:

$$
\text { Degree of alkylation }(\%)=\{(\mathrm{A} / 3) /(\mathrm{B} / 4)\} \times 100
$$

\subsection{Emulsification}

The emulsification of PGA alkylates was measured as described by Dong et al. with some modifications [12]. 
The PGA alkylate was dissolved in $4.5 \mathrm{ml}$ of MilliQ water (0.02\%) in a glass vial. $0.5 \mathrm{ml}$ of clove oil was added and the vial was shaken at $110 \mathrm{rpm}$ for $30 \mathrm{~min}$ at $28^{\circ} \mathrm{C}$. Optical density (OD) was measured at $530 \mathrm{~nm}$ immediately after shaking. To evaluate the emulsion-stabilizing activity, OD was measured sequentially at $10,20,30$, 60, 90, and 120 min of standing after shaking. Commercial emulsifier and emulsion stabilizer were also measured as controls.

\subsection{Moisture-Absorption and -Retention Capacities}

The moisture-absorption and -retention capacities were measured as described by Urai et al. [7].

\section{Results and Discussion}

\subsection{Chemical Properties of PGA Alkylates}

Using the various alkyl bromides, PGA was successfully alkylated. Figure 1 shows the ${ }^{1} \mathrm{H}$ NMR spectra and chemical structures of the raw materials, PGA and 1-bromohexadecane, and the synthesized PGA hexadecanoate.

(a)<smiles>CCC(NC)C(=O)O</smiles>

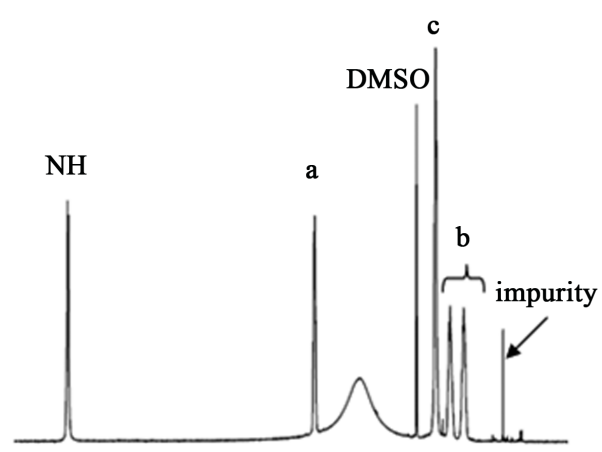

(b)

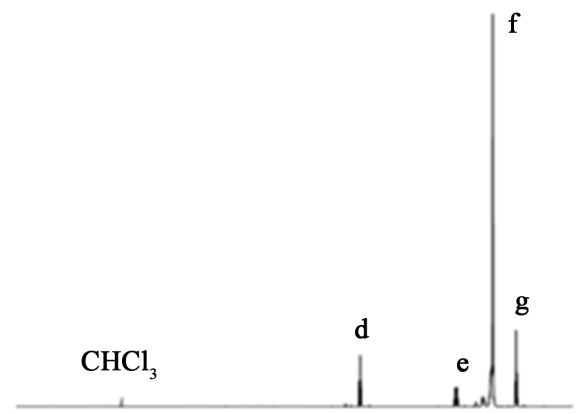

(c) $\begin{array}{cccr}\text { d } & \text { e } & \text { f } & \text { g } \\ \mathrm{Br}-\mathrm{CH}_{2} & -\mathrm{CH}_{2} & -\left(\mathrm{CH}_{2}\right)_{13} & -\mathrm{CH}_{3}\end{array}$

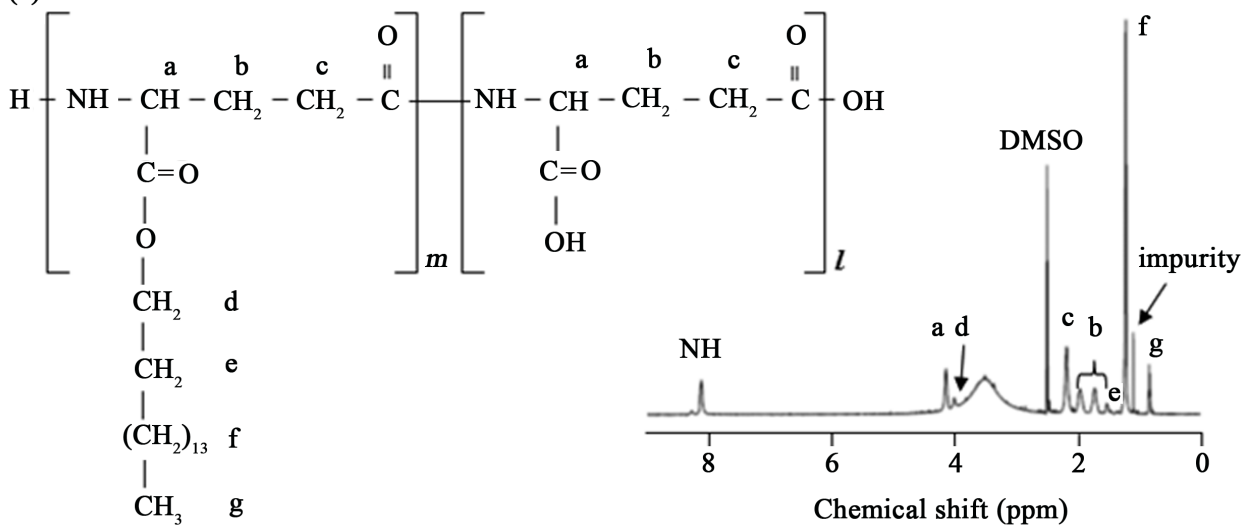

Figure 1. ${ }^{1} \mathrm{H}$ NMR spectra and structures of PGA (a), 1-bromohexadecane (b), and hexadecanoyl ester of PGA (c). ${ }^{1} \mathrm{H}$ NMR were recorded in DMSO- $d_{6}(\mathrm{~A}, \mathrm{C})$, or $\mathrm{CDCl}_{3}(\mathrm{~B})$ at $40^{\circ} \mathrm{C}$. 
The signal of the methylene proton bound to the bromo atom in 1-bromohexadecane (Figure 1(b) and Figure 1(d)) is downfield to that in the PGA hexadecanoate spectrum (Figure 1(c) and Figure 1(d)). This indicates that the $\alpha$-carboxyl residue of PGA is esterified by the hexadecanoyl group [13]. PGA alkylation was also confirmed by 2D NMR analysis (data not shown).

The degree of alkylation is controlled by changing the concentration of the alkyl bromide in the alkylation reaction (Figure 2). At the same concentration, however, a greater chain length leads to a higher degree of alkylation, indicating that long-chain alkyl esters are more easily incorporated into PGA than those with shorter chains are. These results suggest that the alkylation method used in this study is effective for synthesizing PGA alkylates with long alkyl chains. Synthesized PGA alkylates were named individually by the length of the alkyl chain and the degree of alkylation. For example, PGA-6-2, indicates that the length of the alkyl chain (carbon number) is six and the degree of alkylation is $2 \%$ (Table 1 ).

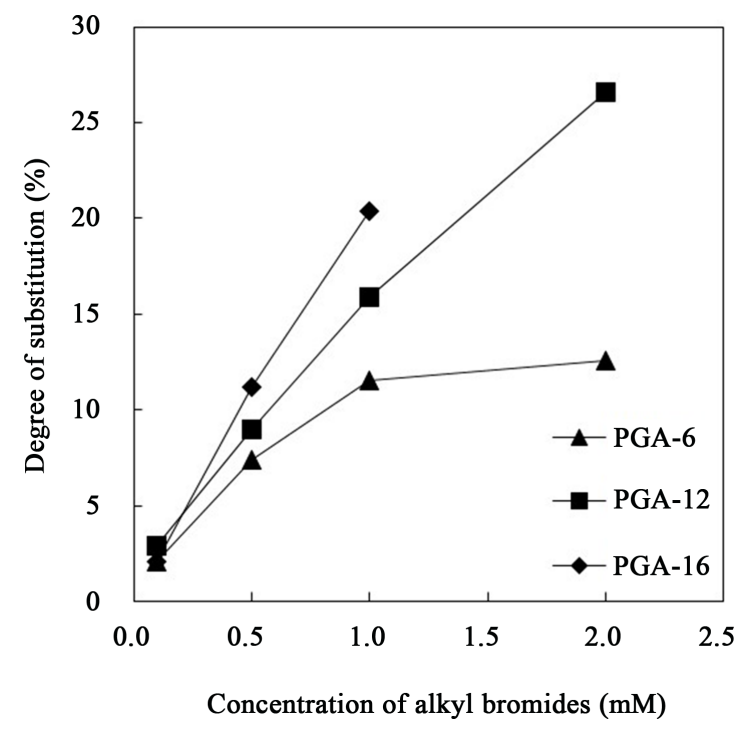

Figure 2. Correlation of the alkyl bromide concentration used in the reaction and the degree of substitution of the PGA ester products.

Table 1. Chemical properties of PGA and synthesized PGA alkylates.

\begin{tabular}{ccccc}
\hline Sample name & $\begin{array}{c}\text { Length of alkyl chain } \\
\text { (carbon number) }\end{array}$ & Degree of alkylation (\%) $)^{\text {a) }}$ & \multicolumn{2}{c}{ Solubility $^{\text {b) }}$} \\
\hline PGA & 0 & 0 & - & + \\
PGA-6-2 & 6 & 2 & + & + \\
PGA-6-7 & 6 & 7 & + & + \\
PGA-6-12 & 6 & 12 & + & + \\
PGA-6-13 & 6 & 13 & + & + \\
PGA-12-3 & 12 & 3 & + & + \\
PGA-12-9 & 12 & 9 & + \\
PGA-12-16 & 12 & 16 & + \\
PGA-12-27 & 12 & 27 & + & + \\
PGA-16-2 & 16 & 2 & + & + \\
PGA-16-11 & 16 & 11 & + & + \\
PGA-16-20 & 16 & 20 & + \\
\hline
\end{tabular}

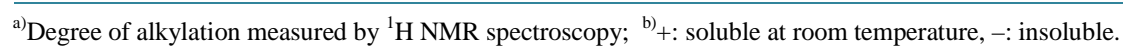


We examined the solubility of native PGA and PGA alkylates in MilliQ water or DMSO (Table 1). The native PGA is insoluble in MilliQ water, but the synthesized PGA alkylates are water-soluble, except for PGA-1620 , which is soluble in DMSO. The low solubility of native PGA in water may be due to the very strong intermolecular interactions caused by hydrogen bonding of the carboxyl groups [13]. In contrast, the hydrogen bonding between the PGA alkylates may be inhibited by the alkyl chains, making them soluble in water. In the case of PGA-16-20, the high degree of long-chain alkylation may increase the hydrophobicity of the PGA, making it insoluble in water.

\subsection{Characterization of the Emulsification of PGA Alkylates}

The emulsification of PGA alkylates was examined using clove oil. Figure 3 shows the relationship between the OD (emulsifying ability) and the degree of substitution of alkyl esters with different chain lengths. The emulsifying ability of PGA is improved by alkylation with longer chains. PGA-12 and PGA-16 have a high OD at a low degree of substitution, whereas PGA-6 needs a higher degree of substitution to achieve high OD values. Since the hydrophobic interaction between the hexanoyl ester of PGA-6 and the surface of the oil is weak, a high degree of substitution is necessary to form a good emulsion. The series of PGA-12 consistently has higher OD values than the PGA-16 series. This result suggests that dodecanoyl is the most suitable ester for high emulsification, which is due to the hydrophobic-hydrophilic balance.

We also examined the emulsion-stabilizing ability of the PGA alkylates. Figure 4 shows the change in OD over time of PGA-12-9, which has the highest emulsification ability within the series of PGA alkylates. PGA12-9 shows the highest OD value just after shaking compared to that of the commercial emulsifier (sodium cholate) and the emulsion stabilizer (gelatin), and the high OD is maintained after $120 \mathrm{~min}$ of standing after shaking. The entire series of PGA alkylates also shows strong emulsion-stabilizing activity (data not shown). The electronic repulsion and intermolecular force between PGA chains are important to stabilize the emulsion.

Figure 5 summarizes the predicted model of the emulsifying and emulsion-stabilizing mechanisms of the PGA alkylates.

In general, low molecular-weight emulsifiers have a strong emulsifying ability, while the emulsion-stabilizing ability is low. In the food industry, polysaccharides or gelatin are added with to the emulsion as stabilizers. Since the PGA alkylates have strong emulsifying and emulsion-stabilizing ability in one molecule, it can be applied to various industries.

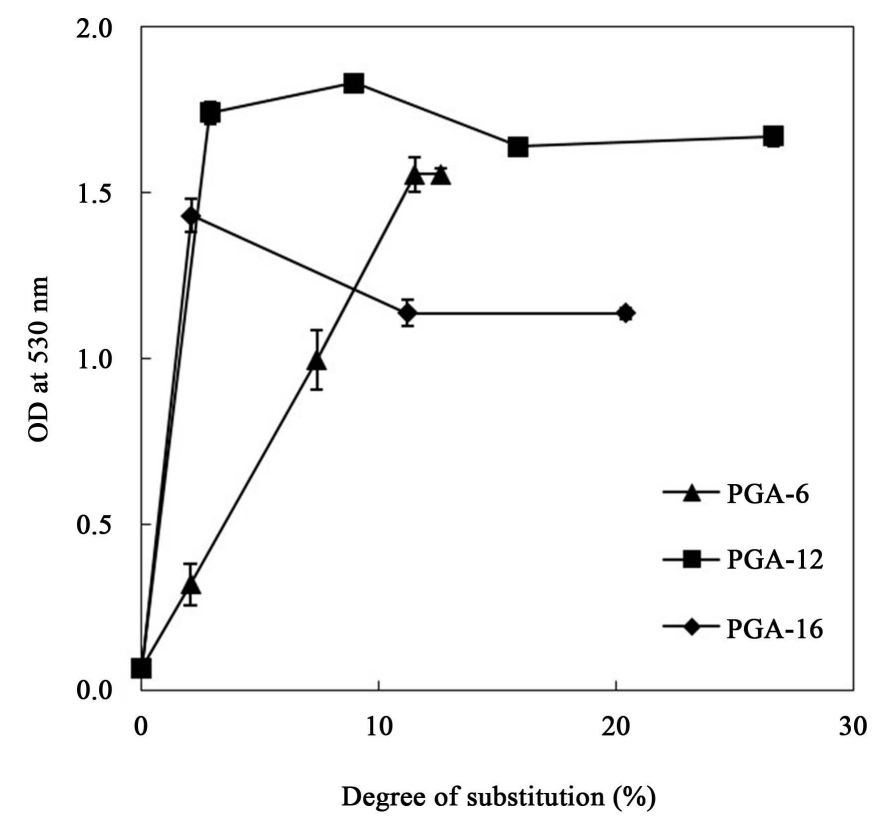

Figure 3. Relationship between the optical density (OD) (emulsifying ability) and the degree of substitution of PGA alkyl esters with different chain lengths. 


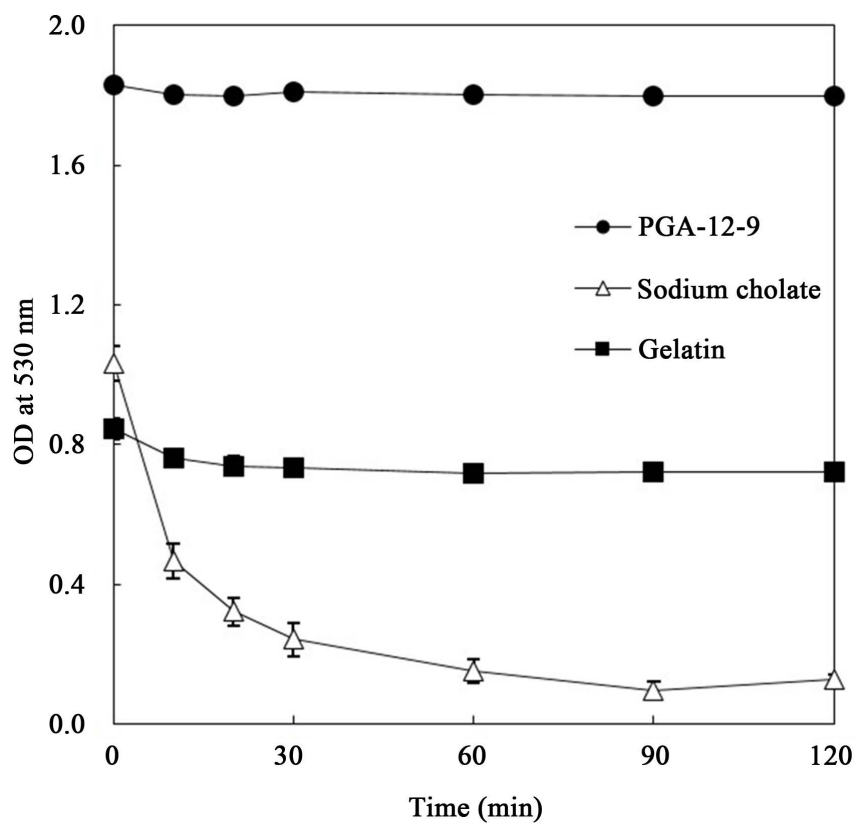

Figure 4. Emulsion-stabilizing ability of the PGA alkylate, PGA-12-9, compared with other emulsion stabilizers (sodium cholate and gelatin).

(a)

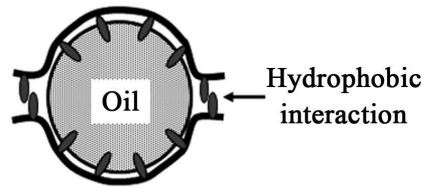

(b)

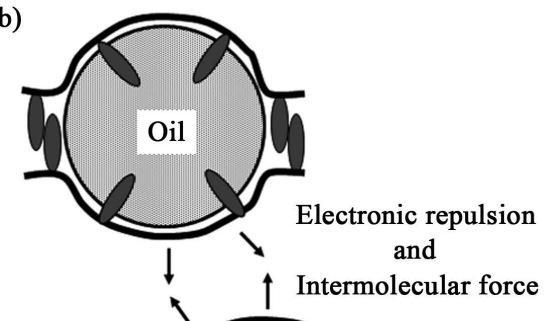

(c)

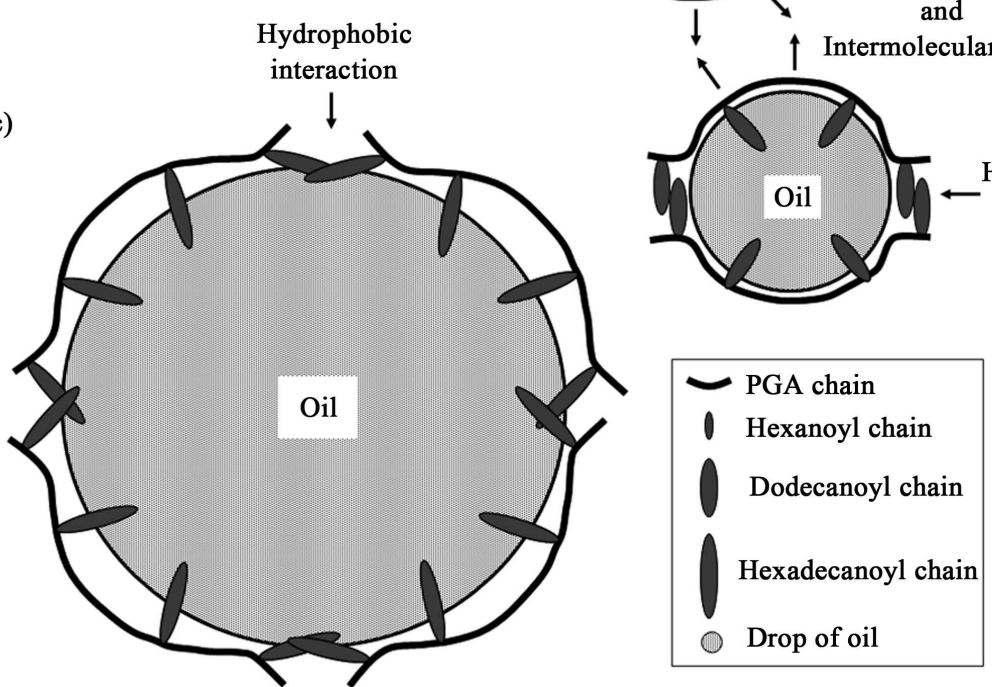

Figure 5. One predicted model of the emulsification and emulsion-stabilizing mechanism of PGA alkylates. (a) PGA alkylate with hexanoyl ester (PGA-6): The hydrophobic interaction between the hexanoyl ester and the oil surface is weak. It needs a high degree of alkylation to form a good emulsion; (b) PGA alkylate with dodecanoyl ester (PGA-12): The hydrophobic interaction between the dodecanoyl ester and the oil surface is suitable for forming a good emulsion. The electronic repulsion and intermolecular force between PGA chains are important to stabilize the emulsion; (c) PGA alkylate with hexadecanoyl ester (PGA-16): The hydrophobic interaction caused by hexadecanoyl ester is too strong to form a good emulsion. Because the size of emulsion droplets formed by PGA-16 is larger than that of PGA-12, it showed a low optical density value. 


\subsection{Characterization of Moisture Absorption Capacities of PGA Alkylates}

Figure 6 shows the relationship between the moisture absorption ability and the degree of substitution of PGA alkylates with different chain lengths, under $47 \%$ relative humidity. The moisture absorption ability of PGA is improved by a low degree of substitution, while this ability decreases with a high degree of substitution. The ability also decreases with the increase in the chain length of the alkyl ester. The highest absorption ability of the PGA alkylates is found in PGA-6-2, which absorbs $11.8 \%$ of its initial weight in moisture. This ability is almost equal to commercially available moisture-absorbents, such as glycerol (12.8\%) and silica gel (13.4\%).

Figure 7 shows the predicted model of the moisture absorption mechanism of the PGA alkylates. Native PGA forms strong hydrogen bonds between the PGA chains, as described above, which consumes the sites required

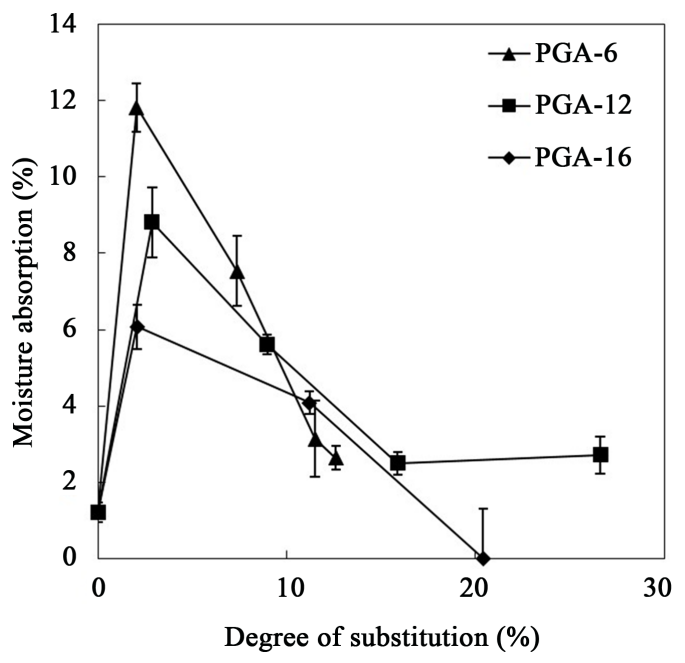

Figure 6. Relationship between the moisture-absorbing ability and the degree of substitution of PGA alkyl esters with different chain lengths.

(a)

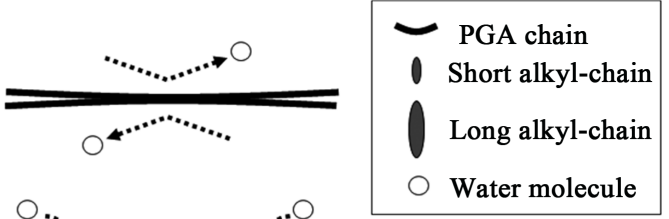

(b)

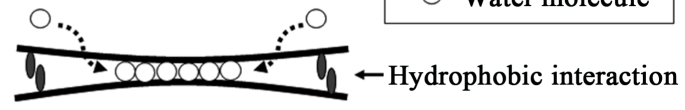

(c)

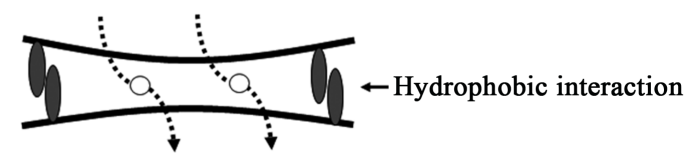

(d)

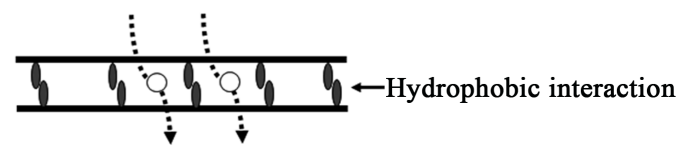

Figure 7. One predicted model of the moisture absorption mechanism of PGA alkylates. (a) Native PGA: Strong hydrogen bonding between the PGA chains prevents moisture absorption; (b) PGA alkylates having a low degree of alkylation with short alkyl chains: Moderate interspaces form between the molecules, which is suitable for trapping water molecules; (c) PGA alkylates having a low degree of alkylation with long alkyl chains: Interspaces that are too large to trap water molecules are formed; (d) Highly alkylated PGA: The number of carboxyl groups is not enough to trap many water molecules. Interspaces that are too large to trap water molecules are formed. 
for forming hydrogen bonds with water molecules. On the other hand, PGA alkylates having a low degree of alkylation form moderate interspaces between the PGA chains due to the presence of the alkylate groups; these interspaces may be suitable to trap water molecules. However, since the carboxyl group of PGA is important for trapping water molecules by hydrogen bonding, a high degree of alkylation, which reduces the availability of the $\alpha$-carboxyl groups, can attenuate the moisture-absorbing ability (Figure 6). For highly alkylated PGAs, the number of carboxyl groups is insufficient to trap many water molecules. Moreover, the interspaces may be too large to trap water molecules. Thus, a low degree of alkylation is most effective for high moisture-absorption in PGA, since hydrogen bonding between PGA molecules is inhibited and the carboxyl groups are available to trap water molecules.

The chain length of the alkyl ester is also an important factor for the moisture-absorbing ability. The ability decreases with an increase in the chain length of the alkyl ester. This result indicates that the long-chain alkyl ester expands the interspace between PGA chains compared to the shorter ones. This form may be a disadvantage for moisture absorption, since the distance of the carboxyl groups between the PGA chains is too far to trap water molecules.

\subsection{Characterization of Moisture Retention Capacities of PGA Alkylates}

Figure 8 shows the relationship between the moisture retention ability and the degree of substitution of alkylates with different chain lengths, under 32\% relative humidity. Similar to moisture absorption, the moisture retention of PGA increases when the degree of substitution is low and decreases when the degree of substitution is high. In contrast with the moisture absorption, PGA-12-3 shows the highest moisture retention ability in the PGA alkylates (66.9\%), almost equal to glycerol (51.1\%) and silica gel (59.9\%).

Figure 9 shows the predicted model of the moisture retention mechanism of PGA alkylates. The mechanism of moisture retention in the PGA alkylates seems similar to that of moisture absorption because this ability decreases with an increase in the degree of alkylation. However, in contrast to the moisture-absorbing ability, PGA-12-3 shows the highest moisture-retention ability compared to the other PGA alkylates. During the moisture retention measurement, drops of water were added to the solid PGA alkylates. In the case of PGA alkylates having a low degree of alkylation with hexanoyl and dodecanoyl esters, a "water-in-oil" type emulsion is formed between the water and air phases, which may prevent the evaporation of added water. In particular, the hydrophobic interactions caused by the dodecanoyl ester greatly expand the interspace between PGA alkylates, causing a strong retention of the added water. On the other hand, the hydrophobic interaction between the PGA hexadecanoate is too strong to expand the interspace between PGA alkylates, thus preventing the penetration of

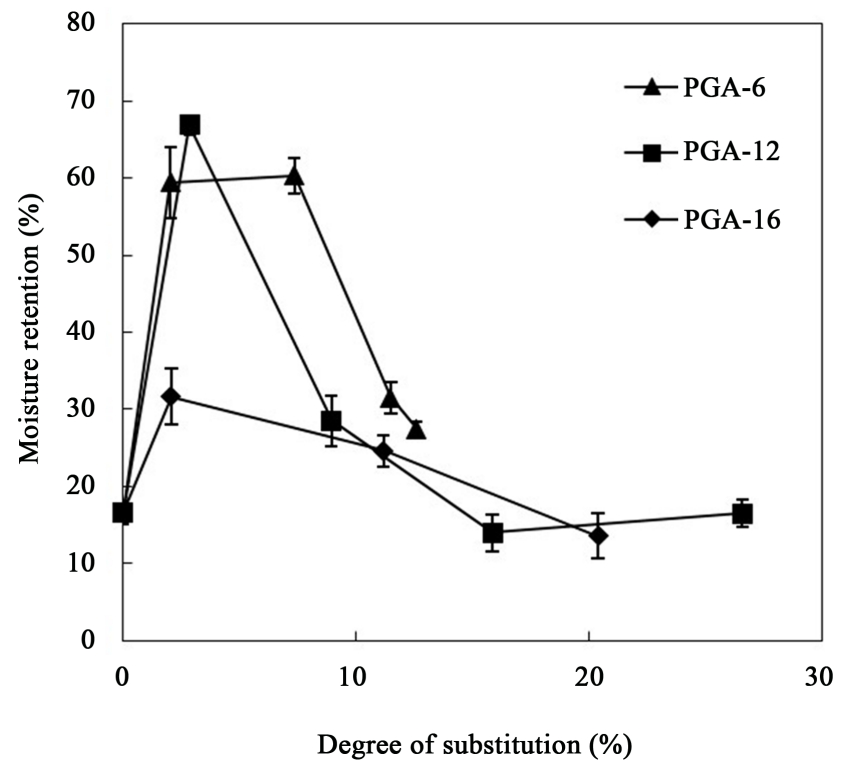

Figure 8. Relationships between the moisture retention ability and the length and degree of substitution of PGA alkyl esters with different chain lengths. 
(a)

Evaporation of added water

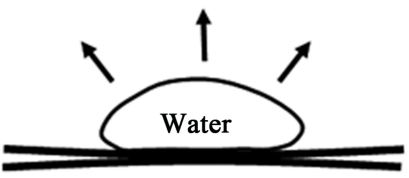

(b)

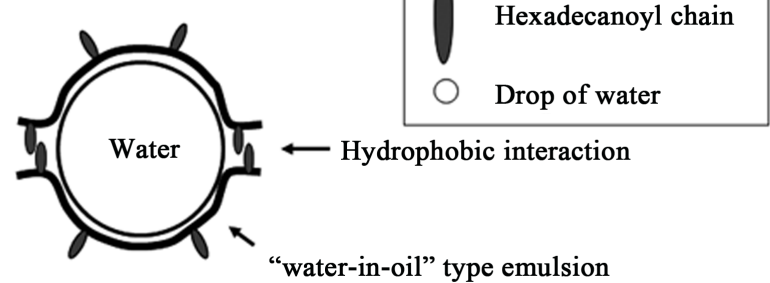

(c)

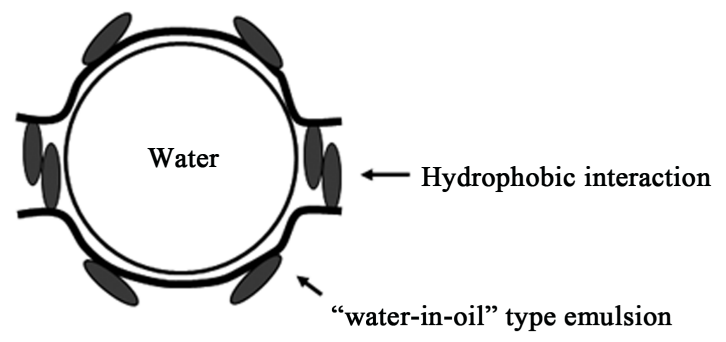

(d)

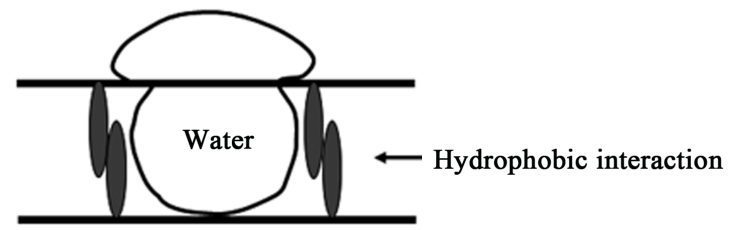

(e)

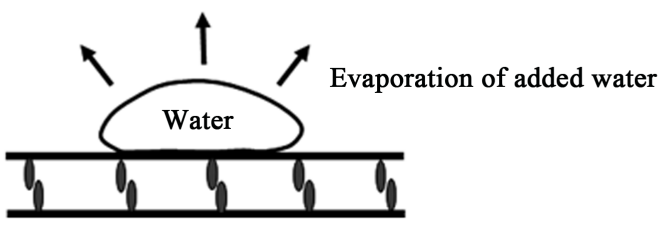

Figure 9. One predicted model of the moisture retention mechanism of the PGA alkylates. (a) Native PGA: Strong hydrogen bonding between PGA chains prevents the penetration of added water; (b) PGA alkylates having a low degree of alkylation with hexanoyl ester: Moderate interspaces are formed, which retain the added water as "water-in-oil" type emulsion; (c) PGA alkylates having a low degree of alkylation with dodecanoyl ester: Hydrophobic interactions caused by dodecanoyl ester greatly expand the interspace between PGA alkylates, and it retains the added water strongly; (d) PGA alkylates having a low degree of alkylation with hexadecanoyl ester: Hydrophobic interactions caused by hexadecanoyl ester are too strong to expand the interspaces between PGA alkylates, preventing the penetration of much water; (e) Highly alkylated PGA: The high degree of alkylation provides strong hydrophobic interactions between the PGA alkylates, which prevents the penetration of added water.

much water. The highly alkylated PGA may also generate a strong hydrophobic interaction between the PGA alkylates, which prevents penetration of added water.

Highly efficient water-absorbing petrochemical polymers are classified chemically as (1) polyelectrolytes, (2) cross-linked, and (3) polymeric [14]. The PGA alkylate is an anionic polymer with some hydrophobic residues that provide a hydrophobic interaction between the PGA alkylate chains, which may cause it to behave as a cross-linked polymer. The hydrophobic-hydrophilic balance, in addition to the number of sites available to form hydrogen bonds with water molecules, may be important to improve the moisture retention ability of PGA alkylates. 


\section{Conclusion}

We showed that alkylation improves the emulsification, moisture absorption, and moisture retention abilities of PGA. To improve the moisture absorption of PGA, a low degree of alkylation with a short chain length is effective in forming interspaces between PGA chains to trap water molecules. Hydrophobic-hydrophilic balance may also be important to improve the emulsification and moisture retention abilities of PGA alkylates. To the best of our knowledge, this is the first report of the relationships between the structure and the multifunctional abilities of an anionic polymer incorporated with a small amount of hydrophobic residues. PGA alkylates, as well as fattyacid-containing polysaccharides, have a potential use as multifunctional surfactants in various industries, e.g., cosmetics or pharmaceuticals.

\section{Acknowledgements}

We are most grateful to T. Beppu for giving us the opportunity to complete this research. We thank K. Ueda at the Institute of Applied Life Science for use of the instrumental facilities. We also thank T. Fukuhara for his valuable discussion. We acknowledge the Integrated Research Institute for use of its NMR facilities. We also acknowledge M. Suzuki, K. Shimoda, and various members of our laboratories for their suggestions, encouragement, and technical assistance. This study was supported in part by grants from the Takano Life Science Research Foundation (Japan).

\section{References}

[1] Iwabuchi, N., Sunairi, M., Urai, M., Itoh C., Anzai, H., Nakajima, M. and Harayama, S. (2002) Extracellular Polysaccharides of Rhodococcus rhodochrous S-2 Stimulate the Degradation of Aromatic Components in Crude Oil by Indigenous Marine Bacteria. Applied and Environmental Microbiology, 68, 2337-2343. http://dx.doi.org/10.1128/AEM.68.5.2337-2343.2002

[2] Urai, M., Anzai, H., Ogihara, J., Iwabuchi, N., Harayama, S., Sunairi, M. and Nakajima, M. (2006) Structural Analysis of an Extracellular Polysaccharide Produced by Rhodococcus rhodochrous Strain S-2. Carbohydrate Research, 341, 766-775. http://dx.doi.org/10.1016/j.carres.2005.12.013

[3] Aizawa, T., Neilan, B., Couperwhite, I., Urai, M., Anzai, H., Iwabuchi, N., Nakajima, M. and Sunairi, M. (2005) Relationship between Extracellular Polysaccharide and Benzene Tolerance of Rhodococcus sp. 33. Actinomycetologica, 19, 1-6. http://dx.doi.org/10.3209/saj.19.1

[4] Urai, M., Aizawa, T., Anzai, H., Ogihara, J., Iwabuchi, N., Neilan, B., Couperwhite, I., Nakajima, M. and Sunairi, M. (2006) Structural Analysis of an Extracellular Polysaccharide Produced by a Benzene Tolerant Bacterium, Rhodococcus sp. 33. Carbohydrate Research, 341, 616-623. http://dx.doi.org/10.1016/j.carres.2006.01.010

[5] Urai, M., Yoshizaki, H., Anzai, H., Ogihara, J., Iwabuchi, N., Harayama, S., Sunairi, M. and Nakajima, M. (2007) Structural Analysis of an Acidic, Fatty Acid Ester-Bonded Extracellular Polysaccharide Produced by a PristaneAssimilating Marine Bacterium, Rhodococcus erythropolis PR4. Carbohydrate Research, 342, 933-942. http://dx.doi.org/10.1016/j.carres.2007.02.001

[6] Urai, M., Yoshizaki, H., Anzai, H., Ogihara, J., Iwabuchi, N., Harayama, S., Sunairi, M. and Nakajima, M. (2007) Structural Analysis of Mucoidan, an Acidic Extracellular Polysaccharide Produced by a Pristane-Assimilating Marine Bacterium, Rhodococcus erythropolis PR4. Carbohydrate Research, 342, 927-932. http://dx.doi.org/10.1016/j.carres.2007.02.002

[7] Urai, M., Anzai, H., Iwabuchi, N., Sunairi, M. and Nakajima, M. (2002) A Novel Moisture-Absorbing Extracellular Polysaccharide from Rhodococcus rhodochrous SM-1. Actinomycetologica, 16, 26-31.

http://dx.doi.org/10.3209/saj.16_26

[8] Urai, M., Anzai, H., Iwabuchi, N., Sunairi, M. and Nakajima, M. (2004) A Novel Viscous Extracellular Polysaccharide Containing Fatty Acids from Rhodococcus rhodochrous ATCC 53968. Actinomycetologica, 18, 15-17. http://dx.doi.org/10.3209/saj.18_15

[9] Shih, I.L. and Van, Y.T. (2001) The Production of Poly-(gamma-glutamic acid) from Microorganisms and Its Various Applications. Bioresource Technology, 79, 207-225. http://dx.doi.org/10.1016/S0960-8524(01)00074-8

[10] Kunioka, M. (1997) Biosynthesis and Chemical Reactions of Poly(amino acid)s from Microorganisms. Applied Microbiology and Biotechnology, 47, 469-475. http://dx.doi.org/10.1007/s002530050958

[11] Kubota, H., Nambu, Y. and Endo, T. (1995) Convenient Esterification of Poly( $\gamma$-glutamic acid) Produced by Microorganism with Alkyl Halides and Their Thermal Properties. Journal of Polymer Science Part A: Polymer Chemistry, 33, 85-88. http://dx.doi.org/10.1002/pola.1995.080330110 
[12] Dong, C. and Tian, B. (1999) Studies on Preparation and Emulsifying Properties of Guar Galactomannan Ester of Palmitic Acid. Journal of Applied Polymer Science, 72, 639-645.

http://dx.doi.org/10.1002/(SICI)1097-4628(19990502)72:5<639::AID-APP4>3.0.CO;2-K

[13] Shimokuri, T., Kaneko, T., Serizawa, T. and Akashi, M. (2004) Preparation and Thermosensitivity of Naturally Occurring Polypeptide Poly( $\gamma$-glutamic acid) Derivatives Modified by Propyl Groups. Macromolecular Bioscience, 4, $407-$ 411. http://dx.doi.org/10.1002/mabi.200300073

[14] Buchholz, F.L. and Peppas, N.A. (1994) Superabsorbent Polymers Science and Technology. ACS Symposium Series, 573, 160p. http://dx.doi.org/10.1021/bk-1994-0573 